\title{
Computation of Trust Value Based on Influence
}

\author{
Mao XinYu \\ Computer School of Wuhan University \\ Wuhan (430072), Hubei, China \\ xinyuchong@sina.com
}

\begin{abstract}
The research of trust management has become a key field as a valid solution to ensure the security and application in p2p networks. The accurate measure of trust is a hard and very important problem in trust management. People's final opinion has been formed based on his own initial opinion with influence of other's opinion in society. In this paper, a new method in computing trust value based on influence is proposed. Learning from the characteristic of people's opinion forming in society, an opinion to a peer (trust or distrust) is also formed on the base of direct trust value with influence of indirect trust value recommended by other peers. By introducing insisting degree and influence function, the influence is quantified. The trust value of a peer is more accurately computed by the method based on influence.
\end{abstract}

Keywords- trust management ,trust value, p2p networks, insisting degree, influence function

\section{INTRODUCTION}

Peer-to-peer (P2P) is a distributed network architecture that provides resource sharing and collaboration between peers. Nowadays, $\mathrm{p} 2 \mathrm{p}$ networks have been becoming one of the most important applications in the Internet [1] [2]. However, the severest problem is the security issue that lies in mutual-trust between two peers, the essentials of openness, freedom, anonymity and loose-coupling relationship among peers in P2P networks lead to a lack of responsibility for the content a peer puts on the network[3] [4]. Moreover, high dynamics of a peer behavior always bring on unsteadiness of $p 2 p$ networks. Therefore, it is necessary to build trust relationship among peers in the distributed large-scale P2P networks [5] [6]. Hence, evaluation of trustworthiness of a participating entity in $\mathrm{p} 2 \mathrm{p}$ networks is more important and key. Currently, there are some approaches of computing trust value or reputation. Such as deterministic approach, deterministic approach, fuzzy logic approach and flow model [7].

The formation of an opinion needs some relevant information about the opinion. In the real world, an opinion of someone is not probably unmistakable due to the limitation of information owned by him in the beginning. Usually, his opinion is gradually formed from a vague attitude to a certain point of view by the influence of friends or other peoples. Therefore, a personal opinion or judgment is often formed on the base of information of his own and under the influence of opinions of others [8] [9]. The formation of an opinion of trust or distrust in $\mathrm{p} 2 \mathrm{p}$ networks is the same as it is in real world. A trust value is computed on the base of direct trust value and under the influence of recommending trust value. Learning from characteristic of opinion forming in human society, the insisting degree is introduced, the influence function is defined and the influence is quantified. The trust value of a peer is more accurately computed. In this paper, trust vale will be computed in the environment of file-sharing network.

The rest of this paper is organized as follow: Section 2 gives some relevant concepts and definitions. Section 3 proposes the computation of trust value based on influence. Section 4 is experiment and analysis. Section 5 is conclusion.

\section{SOME CONCEPTS}

This section gives some relevant concepts and definitions about trust, trust value, direct trust value, insisting degree, and influence function etc.

A. trust and trust value

In this paper, the trust in $\mathrm{p} 2 \mathrm{p}$ networks is defined as follows:

Definition 1 trust: peer $i$ trusting peer $j$ is peer i's subjective expectation of the services provided by peer $\mathrm{j}$ with the peer j's statement.

Trust value (Tval $(i, j))$ is the key quantitative measure of trust. Trust value is usually between 0 and 1.0 means that peer $\mathrm{i}$ fully distrusts peer $\mathrm{j}$. 1 means that peer $\mathrm{i}$ fully trusts peer $\mathrm{j}$.

\section{B. direct trust, direct trust value and recommended trust} value

Definition 2 direct trust: direct trust is a trust relationship that is gradually formed between a peer $i$ (the trustor) and a peer $\mathrm{j}$ (the trustee) with a foundation of the experience in direct transactions.

Direct trust value (DTval) is the quantitative measure of direct trust. DTval $(i, j)$ expresses the direct trust vale of peer $\mathrm{i}$ (the trustor) trusting in peer $\mathrm{j}$ (the trustee).

DTval $(k, j)$ is the direct trust vale of peer $k$ trusting in peer $\mathrm{j}$. For peer i, DTval $(\mathrm{k}, \mathrm{j})$ is the recommended trust value when peer $\mathrm{k}$ recommends the DTval $(\mathrm{k}, \mathrm{j})$ to peer $\mathrm{i}$.

\section{Inisting degree and influence function}

Definition 3 Insisting degree: insisting degree is peer i's the certainty degree of the direct trust between peer $i$ and peer $\mathrm{j}$.

Insisting degree is the characteristic of a process of an opinion forming. The longer time and the higher frequency of interacting between peer $i$ and Peer $j$, the insisting degree 
of the peer i's direct trust in peer $\mathrm{j}$ is higher. On the contrary, the insisting degree is lower.

$\mathrm{U}_{\mathrm{i}}$ denotes the insisting degree of peer i's direct trust in peer $j, n$ is the number of interaction between $i$ and $j, t_{1}$ is the first time of $\mathrm{i}$ interacting $\mathrm{j}$ and $\mathrm{t}_{\text {current }}$ is current time. The following is the formula of insisting degree:

$$
U_{i}=\left(1-\frac{1}{1+\frac{n}{t_{\text {current }}-t_{1}}}\right) \quad 0 \leq \mathrm{Ui} \leq 1
$$

The influence function is given with the definition of insisting degree. Peer $i$ is the trustor. Peer $j$ is the trustee. DTval $(i, j)$ is the direct trust value of peer $i$ to peer $j$. RTval $(\mathrm{k}, \mathrm{j})$ is the recommending trust value of peer $\mathrm{k}$ recommended direct trust value about peer $j$ to peer $i$. $U_{i}$ and $\mathrm{U}_{\mathrm{k}}$ are the insisting degree of $\mathrm{i}$ and $\mathrm{k}$ 's direct trust in $\mathrm{j}$ respectively. The influence function of $\mathrm{k}$ influencing $\mathrm{i}$ is as follows:

$f(i, k)=\left\{\begin{array}{cc}\mathrm{U}_{\mathrm{k}} *\left(\mathrm{U}_{\mathrm{k}}-\mathrm{U}_{\mathrm{i}}\right) & \mathrm{U}_{\mathrm{k}}>\mathrm{U}_{\mathrm{i}} \text { and RTval }(\mathrm{k}, \mathrm{j})>\operatorname{DTval}(\mathrm{i}, \mathrm{j}) \\ -\mathrm{U}_{\mathrm{k}} *\left(\mathrm{Uk}-\mathrm{U}_{\mathrm{i}}\right) & \mathrm{U}_{\mathrm{k}}>\mathrm{U}_{\mathrm{i}} \text { and DTval }(\mathrm{i}, \mathrm{j})>\operatorname{RTval}(\mathrm{k}, \mathrm{j}) \\ 0 & \mathrm{U}_{\mathrm{k}} \leqslant \mathrm{U}_{\mathrm{i}} \text { or DTval }(\mathrm{i}, \mathrm{j})=\operatorname{RTval}(\mathrm{k}, \mathrm{j})\end{array}\right.$

(1) When Uk $>$ Ui and RTval $(k, j)>D T v a l(i, j)$, peer k makes a positive influence on peer $i$. The DTval $(i, j)$ will be raised.

(2) When $U_{k}>U_{i}$ and DTval (i, j)> RTval $(k, j)$, peer k makes a negative influence on peer $i$. The DTval $(i, j)$ will be reduced.

(3) When $U_{k} \leqslant U_{i}$ or DTval $(i, j)=\operatorname{RTval}(k, j)$, k has no influence on $\mathrm{i}$. Peer $\mathrm{i}$ and peer $\mathrm{k}$ insist on their respective opinions when $U_{k}=U_{i} \cdot U_{k}<U_{i}$, it means that the insisting degree of $i$ is greater than the insisting degree of $\mathrm{k}$. Thus $\mathrm{k}$ has no effect on $\mathrm{i}$. DTval $(\mathrm{i}, \mathrm{j})$ $=\operatorname{RTval}(\mathrm{k}, \mathrm{j})$, it means that the opinion of peer $\mathrm{i}$ is the same as peer k's. So the DTval $(i, j)$ has not changed.

\section{TRUST EVALUATING}

Trust evaluating includes computation of direct trust value and trust value [10] [11].

\section{A. Computation of Direct Trust Value}

Direct trust is formed between two peers (such the trustor $i$ and the trustee j) with directly intercommunicating. Direct trust value reflects the trustor's expectation of the trustee. Some parameters are selected for computation of direct trust value.

(1) Feedback Evaluation: feedback evaluation is an important factor in the computation of direct trust value. It is the degree of satisfaction of the trustor $i$ to the services provided by the trustee $\mathrm{j}$ and a subject opinion of the trustee.

(1) Transaction Weight: transaction weight is the importance of a transaction. It is defined by peer or system.
(2) Time Factor: the general principle is that recent transactions make more important role in computation of direct trust vale.

The formula of direct trust value of the peer i (the trustor) trusting in peer $\mathrm{j}$ (the trustee) is as follow:

$$
\text { DTval }(i, j)=\sum_{k=1}^{n} \text { Feval }(i, j)_{k} * W_{k} * T_{k}
$$

Here, $n$ is the total number of transactions, Feval $(i, j)_{k}$ is feedback evaluation of the number $\mathrm{k}$ transaction, $\mathrm{W}_{\mathrm{k}}$ is the transaction weight of the number $\mathrm{k}$ transaction, and $\mathrm{T}_{\mathrm{k}}$ is the time factor of the number $\mathrm{k}$ transaction. The formula of $\mathrm{T}_{\mathrm{k}}$ as follow:

$$
T_{k}=\left(\frac{1}{1+\Delta t}\right)^{n-k} \quad\left(\Delta t=t_{\text {current }}-t_{k}\right)
$$

\section{B. Computation of Trust Value}

Tval $(i, j)$ is the trust value between peer $i$ (the trustor) and peer $j$ (the trustee). DTval $(i, j)$ is the direct trust value between peer $i$ and peer $j$. RTval $(k, j)$ is the recommended trust value of peer $\mathrm{k}$ recommending direct trust value about peer $j$ to peer $i$. $f(i, k)$ is the influence function. The formula of TVal $(i, j)$ is as follows:

$$
\begin{aligned}
& \operatorname{Tval}(i, j)=\operatorname{DTval}(i, j)+f(i, k) * \Delta D R T_{i k} \\
& \Delta D R T_{i k}=|\operatorname{DTval}(i, j)-\operatorname{RTval}(k, j)| \quad, \quad \Delta D R T_{i k} \quad 1 \mathrm{~s} \quad \text { the }
\end{aligned}
$$
difference of opinion.

When many peers recommend their direct trust values about the trustee, the process of computing trust value is as follow:

(1) According to recommending trust values and their insisting degrees, recommending trust values will be divided into the set of positive influence, the set of negative influence and the set of no influence.

(2) The difference of opinion and the influence function are calculated in the set of positive influence and negative influence respectively.

In the set of positive influence (the number of peers is $\mathrm{n}_{1}$ ):

$$
\begin{gathered}
\Delta \operatorname{RTpi}_{i k}=\frac{\sum_{k=1}^{n_{1}} U_{k} *|\operatorname{DTval}(i, j)-R \operatorname{Tval}(k, j)|}{\sum_{k=1}^{n_{1}} U_{k}} \\
f(i, k)_{p i}=\frac{\sum_{k=1}^{n_{1}} U_{k} * U_{k} *\left(U_{k}-U_{i}\right)}{\sum_{k=1}^{n_{1}} U_{k}}
\end{gathered}
$$
$\left.\mathrm{n}_{2}\right)$ :

In the set of negative influence (the number of peers is

$$
\begin{aligned}
\Delta \text { DRTni }_{i k} & =\frac{\sum_{k=1}^{n_{2}} U_{k} * \mid D T v a l(i, j)-R T \text { Tval }(k, j) \mid}{\sum_{k=1}^{n_{2}} U_{k}} \\
f(i, k)_{n i} & =-\frac{\sum_{k=1}^{n_{2}} U_{k} * U_{k} *\left(U_{k}-U_{i}\right)}{\sum_{k=1}^{n_{2}} U_{k}}
\end{aligned}
$$

(3) Calculating trust value:

$\operatorname{Tval}(i, j)=\operatorname{DTval}(i, j)+f(i, k)_{p i} * \Delta D R T p i_{i k}$ 


$$
+f(i, k)_{n i} * \Delta D R T n i_{i k}
$$

\section{EXPERIMENT AND ANALYSIS}

This experiment is to verify the effectiveness of the computation trust value based on influence in the application environment of file-sharing. There are two types peer in this experiment. The quality of services provided by a type of peer is stability. The quality of services provided by another type of peer is uncertainty. A peer selects servicing peer by computing its trust value. Being compared with that of Eigen-trust model [12], the effectiveness of the computation trust value based on influence is analyzed by successful downloading percentage. In figures, I-trust represents the computation of trust value based on influence proposed by this paper.

Condition I : There are 1000 peers. The number of peers providing unstable services is 100 . The number of peers providing stable services is 900 .

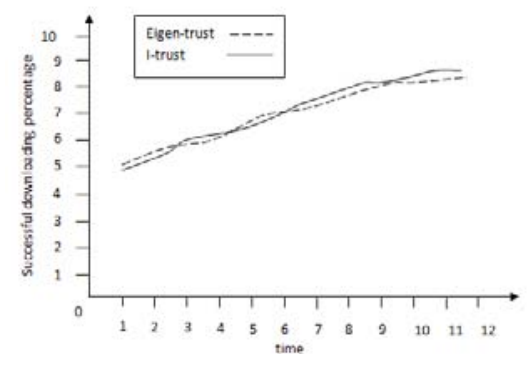

Fig.1 Successful downloading percentage in condition I

In Condition I, most peers provide stable services. The successful downloading percentage of Eigen-trust and I-trust is not much different.

Condition II : There are 1000 peers. The number of peers providing unstable services is 500 . The number of peers providing stable services is 500 .

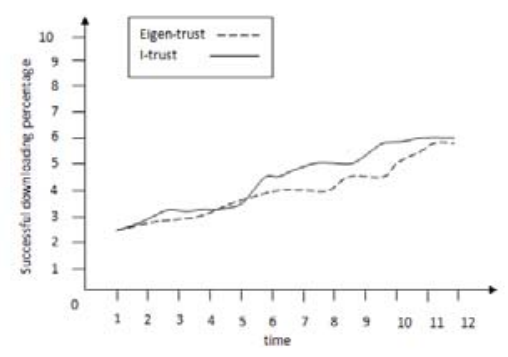

Fig.2 Successful downloading percentage in condition II

In Condition II, the quality of services provided by half of the peers is unstable. On the initial stage, two percentages of successful downloading are little different. On the middle stage, the successful downloading percentage of percentage is significantly higher than that of Eigen-trust. They are approximate on the final stage.

Initial transacting with a providing unstable service peer, feeling of different peers is different. Some may think that its quality of service is good, and some think that its quality of service is poor. A correct opinion will be gradually formed with the number and the frequency of transacting with the providing unstable service peer increasing. The insisting degree is introduced in computation of trust value based on influence. The insisting of degree of an initial trust opinion is lower, and so the direct trust value with lower has little effect on calculating the trust value. A correct trust opinion is gradually formed with frequently transacting, and the insisting degree is increasing at the same time. The direct trust value with higher insisting degree has more effect on calculating the trust value. According to influence function, some recommending trust values with lower insisting degree are abandoned in I-trust. The trust value is more accurate in I-trust than that of Eigen-trust.

\section{CONCLUSION}

Learning from the characteristic of opinion forming in human society, computation of trust value based on influence is proposed. Some recommending trust values are abandoned by their insisting degree and influence function. The recommending trust value with higher insisting degree has more effect on calculating the trust value. The recommending trust value with lower insisting degree has little effect, even no effect on calculating the trust value. The trust value is accurately calculated in the environment in which behavior of many peers is unstable.

\section{REFERENCE:}

[1] Sini Ruohomaa and Lea Kutvonen Trust management survey P.Herrmann et al. (Eds): iTrust 2005, LNCS 3477, pp. 77 - 92

[2] R.Krishnan, M.D.Smith and RTelang. "The Economics of Per-ToPeer Networks". Journal. Of Information Technology Theory and Applications5 (3):2003,3 1.44

[3] T. Beth, M Borcherding, B. Klien. Valuation of trust in open network[C]. In Proc of the European Symp on Research in Security (ESORICS). Berlin: Springer-Verlag, 1994. 3-18

[4] Azzedin F, Maheswaran M. Evolving and managing trust in grid computing systems Electrical and Computer Engineering, IEEE CCECE 2002 Canadian

[5] Povey D. Developing electronic trust policies using a risk management model [A]. Proceedings of the 1999 CQRE Congress.

[6] A JØsang. A Metric for trusted systems[C]. Wien: Austrian Computer Society, 1998. 541-549

[7] Chithra Selvaraj, Sheila Anand. A survey on Security Issues of Reputation Management Systems for Peer-to-Peer Networks[J]. Computer Science Review 6(2012) Elservier Inc. 2012.04

[8] Moge P R , Noshir C. Theories of Communication Networks[M]. NY: Oxford University Press. 2003

[9] Luo JiaDe. The handout of Social Networks Analysis[M]. Beijing: Sciences Academic Press. 2005

[10] Azzedin F, Maheswaran M. A trust brokering system and its application to resource management in public resource grids 18th International Parallel and Distributed Processing Symposium (IPDPS'04) C. Santa Fe :IEEE Computer Society, 2004.

[11] T.Grandison and M.sloman. A survey of trust in internet application. IEEE Communications Survey and Tutorials, 2000, Vol.4 (4):2-16

[12] Kamvar S D, Schlosser M T, Garcia-Molina H. The Eigen trust Algorithm for Reputation Management in P2P Networks[C]. Proceeding of the $12^{\text {th }}$ International World Wide Web Conference. Budapest, Hungary: ACM Press, 2003 